\title{
Water- versus land-based exercise in elderly subjects: effects on physical performance and body composition
}

This article was published in the following Dove Press journal:

Clinical Interventions in Aging

26 August 2013

Number of times this article has been viewed

\author{
Marco Bergamin' \\ Andrea Ermolao' \\ Silvia Tolomio' \\ Linda Berton ${ }^{2}$ \\ Giuseppe Sergi \\ Marco Zaccaria' \\ 'Sports Medicine Division, ${ }^{2}$ Geriatrics \\ Division, University of Padova, \\ Padua, Italy
}

\begin{abstract}
The aim of this study was to assess the effectiveness of a 24 -week exercise protocol carried out in geothermal spring water to improve overall physical function and muscle mass in a group of healthy elderly subjects. A further aim was to compare this water-based protocol with a land-based protocol and a control group. For this purpose, 59 subjects were recruited and randomly allocated to three groups: aquatic group (AG), land group (LG), and control group (CG). AG and LG followed a 6-month, twice-weekly, multimodality exercise intervention. AG underwent the protocol in hot-spring water $\left(36^{\circ} \mathrm{C}\right)$ while $\mathrm{LG}$ did it in a land-based environment. After the intervention, knee-extension strength was maintained in AG and LG. The 8-foot upand-go test showed a reduction in both exercise groups (AG $-19.3 \%, P<0.05$; LG $-12.6 \%$, $P<0.05)$, with a significantly greater decrease in AG. The back-scratch test revealed an improvement only in AG $(25.8 \% ; P<0.05)$, while the sit-and-reach test improved in all groups. Finally, AG reduced fat mass by $4 \%(P<0.05)$, and dominant forearm fat decreased by $9.2 \%$ $(P<0.05)$. In addition, calf muscle density increased by $1.8 \%(P<0.05)$. In summary, both water- and land-based activities were beneficial in maintaining strength and in improving lower-body flexibility. Aquatic exercise appeared a better activity to improve dynamic balance. Thermal swimming pools and the use of rating of perceived exertion as a method of exercise monitoring should be considered potentially useful tools to enhance physical performance and body composition in healthy elderly.
\end{abstract}

Keywords: aging, multimodality exercise, performance, muscle mass

\section{Introduction}

Sarcopenia has been defined as the loss of muscular mass and decline in associated muscular function occurring with aging. Its development and progression is a complex and multifactorial process, resulting from changes only partially understood. ${ }^{1}$ A growing body of evidence emphasizes how physical activity ${ }^{2}$ or other types of interventions ${ }^{3}$ or their combination ${ }^{4,5}$ can slow the loss of skeletal mass. From a clinical point of view, physical performance and muscle strength were also suggested as first indexes of sarcopenia and thus emphasized as primary outcome domains for intervention trials in sarcopenia. ${ }^{6}$

Aquatic activities could be included as a strategy to counteract physical decline. Recent studies have investigated the effects of water-based exercise, though mainly in specific clinical conditions, such as coronary artery disease, ${ }^{7,8}$ fibromyalgia, ${ }^{9,10}$ lowback pain ${ }^{11,12}$ and low bone mineral density. ${ }^{13}$

Although it appears that water-based exercise may be a suitable exercise modality for elderly individuals, little is known about its ability to improve body composition
Correspondence: Marco Bergamin Sports Medicine Division, Department of Medicine, University of Padova,

Via Giustiniani 2, Padova, Italy

Tel +3904982I 5763

Fax +3904982I 5862

Email marco.bergamin@unipd.it 
and muscle mass in older adults who regularly engage in these activities. Recently, a paper reviewed the effects of exercise in an aquatic setting on physical fitness and body composition in healthy elderly, ${ }^{14}$ and highlighted the need to adopt more advanced techniques to measure muscle mass, which are currently lacking.

Another literature gap regards the use of geothermal spring water as an exercise setting, particularly in old individuals. The warmth of the water may enhance blood flow, which is thought to help in dissipating algogenic chemicals and facilitating muscle relaxation. ${ }^{15}$ However, not much data exist about the effectiveness and safety of exercise protocols carried out in this environment.

Our aim was to assess the effectiveness of a 24-week exercise protocol, carried out in hot-spring water, to improve overall physical function and muscle mass in a group of healthy elderly. A further aim was to compare this waterbased protocol with a land-based protocol and a control group.

\section{Methods and materials}

\section{Participants}

Fifty-nine healthy subjects ( 29 male, 30 female) were recruited (age $71.2 \pm 5.4$ years, body mass index $26.5 \pm 3.0 \mathrm{~kg} / \mathrm{m}^{2}$ ) by eight family physicians in the towns of Abano Terme, Montegrotto Terme, Battaglia Terme, and Teolo, settlements around the thermal area of the Euganean Basin (Veneto Region, Italy). For this purpose, advertisements were placed within family physicians' waiting rooms. Information about study purpose and procedure were given to each subject; after acceptance, written consent was obtained before participation. Subjects were randomly allocated to aquatic group (AG), land group (LG), or control group (CG). Table 1 describes sample characteristics at baseline $\left(\mathrm{T}_{0}\right)$ by sex. Figure 1 shows the participants' recruitment and allocation.

The inclusion criteria were age ( $>65$ years) and no exercise contraindications detected from a full physical examination, including cardiovascular, pulmonary, musculoskeletal, and abdominal evaluation. Further, subjects should not have

Table I Baseline characteristics of the study participants (mean \pm standard deviation)

\begin{tabular}{lccr}
\hline & $\begin{array}{l}\text { Male } \\
(\mathbf{n}=\mathbf{2 9})\end{array}$ & \multicolumn{1}{c}{$\begin{array}{l}\text { Female } \\
(\mathbf{n}=\mathbf{3 0})\end{array}$} & \multicolumn{1}{c}{$\begin{array}{l}\text { Total } \\
(\mathbf{n}=\mathbf{5 9})\end{array}$} \\
\hline Age (years) & $72.1 \pm 6.1$ & $72.3 \pm 4.7$ & $71.2 \pm 5.4$ \\
Height $(\mathrm{cm})$ & $169.2 \pm 7.3$ & $156.2 \pm 5.5$ & $162.8 \pm 9.2$ \\
Body mass $(\mathrm{kg})$ & $76.1 \pm 11.3$ & $59.7 \pm 6.5$ & $68.1 \pm 6.5$ \\
BMI $\left(\mathrm{kg} \cdot \mathrm{m}^{-2}\right)$ & $26.6 \pm 3.0$ & $24.5 \pm 2.7$ & $25.6 \pm 2.7$ \\
\hline
\end{tabular}

Abbreviation: BMI, body mass index. attended a structured physical activity or exercise program in the prior 6 months. Other exclusion criteria were represented by a history of central nervous dysfunction such as hemiparesis, myelopathies, cerebral ataxia, significant musculoskeletal deformities (amputation, leg-length inequality, or scoliosis), and abnormalities or arthritis-limited movements by pain. Further exclusion criteria were a clinically evident history of severe cardiovascular disease, which could limit or contraindicate exercise, angina or related symptoms, postural hypotension (defined as fall in arterial blood pressure when changing position of $>20 \mathrm{mmHg}$ in systole or $>10 \mathrm{mmHg}$ in diastole), and use of beta-blockers.

The study complied with the current laws of Italy for research on human participants and was approved by the University Hospital ethical committee.

\section{Materials and procedure}

Functional evaluation was performed at $\mathrm{T}_{0}$ and after the end of the exercise protocol $\left(\mathrm{T}_{1}\right)$. Once the randomization procedure was completed, we performed an evaluation of all control subjects, then participants of the two experimental groups were examined. After 24 weeks from the first evaluation (control subjects), we recalled all controls for the second evaluation. Both experimental groups were evaluated from the beginning of the last week of exercise, and all subjects were tested within 3 weeks.

Body mass was measured using a BWB-800 AS scale (Tanita, Arlington Heights, IL, USA), and height with an HR-200 stadiometer (Tanita). Grip strength was measured by hand-grip dynamometry (Baseline, Elmsford, NY, USA), and isotonic (KET) and isometric knee-extension (KEM) strengths were measured by a dynamometric load cell (Globus Ergo System, Codognè, Italy) applied to a knee-extension device (Technogym, Gambettola, Italy). The 8-foot up-andgo test (UGT) was used to assess dynamic balance while the back-scratch test (BS) and sit-and-reach test (SR) were performed to evaluate upper- and lower-body flexibility. The general procedure of these three field tests has been described and validated elsewhere. ${ }^{16}$ Briefly, the UGT measures the time it takes to get out of a chair, walk 8 feet to and around a cone, and return to the chair; the BS measures the distance between (or the overlap of) the middle fingers behind the back when trying to touch the middle fingers of both hands together behind the back; to perform the SR, subjects sit with the soles of their feet against a box (with a centimeter scale printed on the top surface), and with their hips flexed to about $90^{\circ}$ to assume an upright sitting position. Then, they flex their hip joints and vertebral column to reach forward 


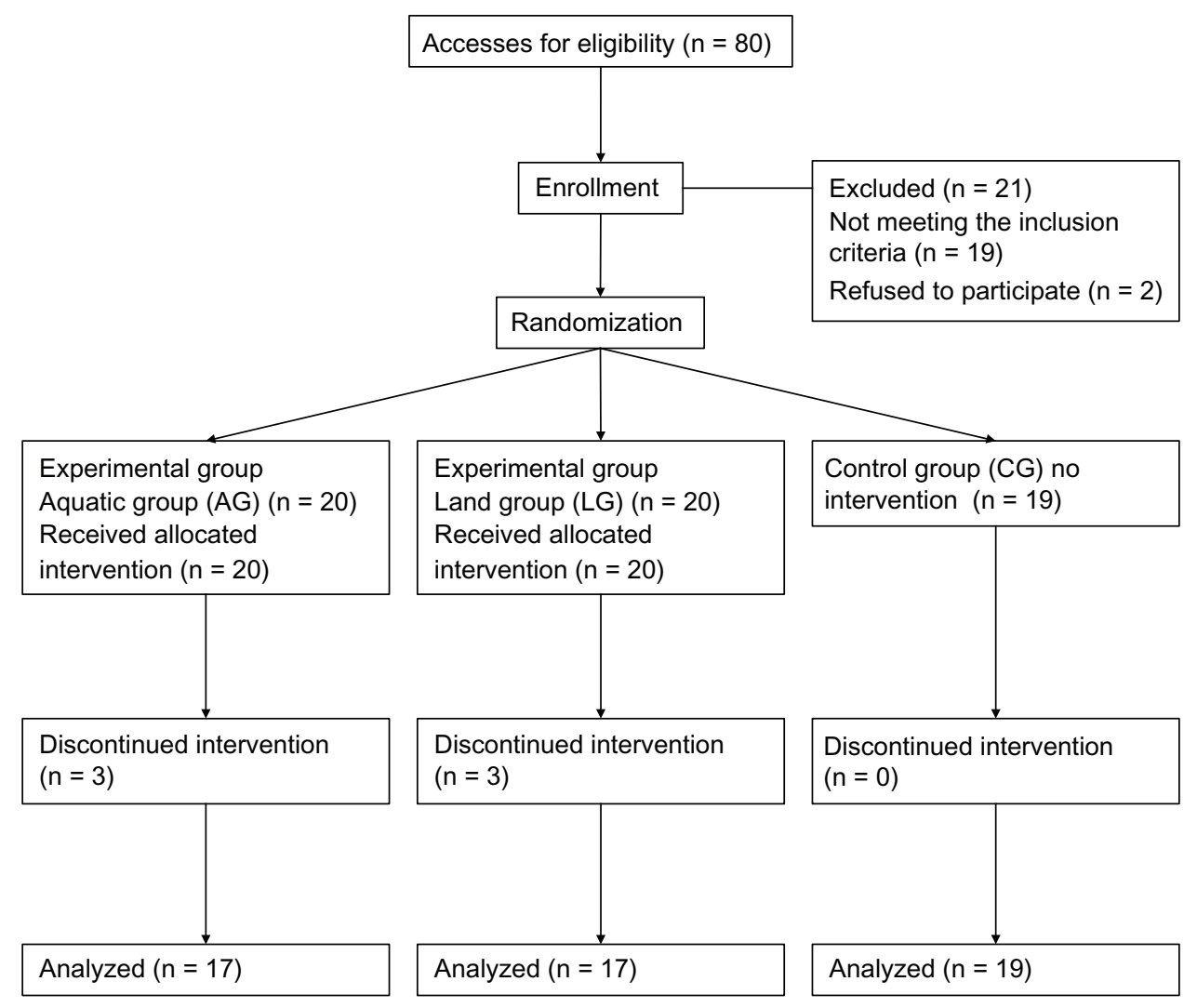

Figure I Recruitment and allocation of the study participants.

as far as possible. The difference (in centimeters) between the starting and the final position is measured. The values included in the statistical analysis represent the mean of the three values for each measure.

Measurements of body composition using the dualenergy X-ray absorptiometry (DXA; QDR $4500 \mathrm{~W}$, software version 12.6; Hologic, Bedford, MA, USA) were performed on all subjects. A standardized procedure for patient positioning and software utilization was used as suggested by the manufacturer $;{ }^{17}$ besides, the accuracy of fat mass (FM) and fat-free mass (FFM) estimates has been examined in a previous validation study. ${ }^{18}$ Appendicular skeletal muscle mass was also calculated following the method of Baumgartner et al. ${ }^{1}$ Peripheral quantitative computed tomography (pQCT; Norland/Stratec XCT-3000, software version 6.0; Stratec Medizintechnik, Pforzheim, Germany) was performed to measure total cross-sectional area, cross-sectional muscle area, and cross-sectional fat area on forearm and on calf. Cross sectional $2.5 \mathrm{~mm}$ scans of the forearm were obtained at $66 \%$ of the radius length from the distal end of the radio. Also for the calf, $2.5 \mathrm{~mm}$ cross-sectional scans were obtained at $66 \%$ of the tibia length from the distal end of the tibia. This position has been described in previous studies on cadavers, demonstrating that this site provides a high estimate of muscle cross-sectional area with direct anatomical measures. ${ }^{19}$ In addition, muscle density was measured, since it has been validated as a reflection of adipose tissue deposition in the muscle..$^{20,21}$ Variation and reliability of muscle density assessed by pQCT has been evaluated elsewhere. ${ }^{22}$ Both DXA and pQCT scans were performed by the same experienced technician.

\section{Exercise interventions}

AG underwent a 6-month twice-weekly exercise protocol in hot-spring water, aimed at improving overall fitness. LG performed an intervention of the same volume, but set in a land-based environment. The exercise trainer was not informed about the aim of the study, but was only asked to follow the specific protocol, as previously determined. The intensity of exercise for both groups was set using the rating of perceived exertion (RPE) ${ }^{23}$ and both groups worked out involving the same muscular groups maintaining the same RPE. Neither AG nor LG used any device to increase drag force or resistance. Training sessions were structured in three parts for both intervention groups (AG and LG) and lasted 60 minutes. The first part (about 8 minutes) included warm-up 
exercises, at very low intensity, such as cervical circumduction, active shoulder, wrist, pelvis, and ankle mobilizations to activate the different body parts, and preparing for the central part of the session. Five lower-body exercises were then performed in a standing position. Exercises were singleleg knee extensions and flexions, hip extensions and flexions (with extended knee), lateral side bounces, calf raises, and lower limb abductions/adductions. After that, five upper-body exercises were carried out: upper-limb abductions/adductions on transverse plane, shoulder flexions/extensions, shoulder abductions/adductions, pushes forward, and lateral pushes. These ten exercises constituted the main part of each session (about 45 minutes). When participants in AG were training the upper body, they were instructed to perform the exercises with knees flexed, to maintain the movement of upper limbs completely underwater. Each exercise lasted 1 minute and was repeated for three times; a 30-second recovery pause was set between each series. The first month of activity was used for exercise familiarization and overall conditioning, maintaining the intensities below 13 in Borg's RPE Scale. The following months, the intensity was set between 13 and 16 RPE, using a progressive incremental protocol, as described in Table 2. Finally, at the end of each training session, about 8 minutes were dedicated to the cool-down, using six stretching positions maintained from 60 to 90 seconds. Muscular regions involved were chest, shoulders, upper and lower back, quadriceps, and hamstrings. Stretching intensity was maintained at moderate intensity, as recommended by American College of Sports Medicine guidelines. ${ }^{24}$ To permit a comparison between aerobic stimulation between $\mathrm{AG}$ and LG, heart rate (HR) was recorded by HR s810i monitors (Polar Electro, Kempele, Finland), and exercise intensity was maintained around $60 \%$ of maximum HR (range 55\%-65\%). The maximum HR was calculated using Cooper's formula $(220$ - age $) .{ }^{25}$ An exercise therapist supervised all the exercise classes. At the beginning of each exercise class, he asked participants to report any problem that had occurred since the end of the last exercise class; he was also instructed to

Table 2 Progression of the 6-month aquatic- and land-based programs

\begin{tabular}{lllll}
\hline Month & Sets & $\begin{array}{l}\text { Exercise time } \\
\text { (sec) }\end{array}$ & $\begin{array}{l}\text { Recovery time } \\
\text { (sec) }\end{array}$ & RPE \\
\hline 1 & 3 & 45 & 45 & $<13$ \\
$2-4$ & 3 & 60 & 30 & $13-14$ \\
$5-6$ & 3 & 60 & 30 & $15-16$ \\
\hline
\end{tabular}

Notes: Exercise and recovery time are expressed in seconds (sec), exercise time indicates the time spent for each exercise set described in the Methods section. Abbreviation: RPE, rate of perceived exertion. record any injury or symptom occurring during the class and reported by participants.

Swimming pool depth range was $1.30-1.80 \mathrm{~m}$; each subject took position where the level of the water reached the medium sternum. The average temperature of the thermal water was $36.2^{\circ} \mathrm{C}$, while land-based activity was performed in a room with a mean temperature of $20.1^{\circ} \mathrm{C}$.

\section{Statistics}

Statistical analyses were carried out using SPSS (version 18.0 for Windows; IBM, Armonk, NY, USA). Results are expressed as means \pm standard deviation or percentage. The Kolmogorov-Smirnov test was carried out to check if data were normally distributed, and Levene's test was performed to test the homogeneity of variance. Student's $t$-test for dependent samples was used to evaluate each variable within groups before versus after exercise intervention. One-way analysis of variance (ANOVA) was adopted to compare the before-and-after differences among the three groups. Finally, post hoc analyses were performed with the Bonferroni correction, to analyze interaction among groups. Significance limits were set at $P<0.05$.

\section{Results}

Among the 59 subjects, only three were smokers, 23 had hypertension, none had type 2 diabetes, and 21 had hypercholesterolemia; 38 were taking medications, mainly represented by antihypertensives, statins, acetylsalicylic acid, benzodiazepine, and non-steroidal anti-inflammatory drugs. Pathologies and the use of medications were distributed between groups. Due to health conditions or lack of interest in participation, six subjects dropped out from the study (three in AG, three in LG, none in CG). The average adherence in the 48 sessions was $81.25 \%$, and no statistically significant differences were detected between AG and LG. During the 6-month protocol, AG and LG aerobic intensities were similar $(P=0.782)$, showing mean values of $60.1 \% \pm 4.6 \%$ (AG) and $59.5 \% \pm 6.6 \%$ (LG) of maximum HR (data gathered during the central part of all exercise sessions).

Tables 3 and 4 show the intragroup differences observed from $\mathrm{T}_{0}$ and $\mathrm{T}_{1}$ in physical function and body composition. When evaluating before-and-after differences among the three groups, ANOVA detected a statistical significance in dynamic balance $(F[2,51]=7.796, P<0.001)$, isotonic leg strength $(F[2,51]=9.624, P<0.001)$, isometric leg strength $(F[2,51]=5.845, P=0.007)$, trunk FM $(F[2,51]=5.541$, $P=0.007)$, total FM $(F[2,51]=19.063, P<0.001)$, trunk FFM $(F[2,51]=5.569, P=0.007)$, and total FFM $(F[2,51]=4.796, P=0.013)$. 
Table 3 Effect of the exercise program on physical performance, upper- and lower-body strength (mean \pm standard deviation)

\begin{tabular}{|c|c|c|c|c|}
\hline & Pre $\left(T_{0}\right)$ & Post $\left(T_{1}\right)$ & $\Delta \%$ & $P$ \\
\hline \multicolumn{5}{|c|}{ Aquatic group $(n=17)$} \\
\hline $\mathrm{BS}(\mathrm{cm})$ & $-7.8 \pm 8.9$ & $-5.81 \pm 8.34$ & 25.8 & 0.042 \\
\hline SR $(\mathrm{cm})$ & $3.2 \pm 7.3$ & $13.08 \pm 6.77$ & 309.9 & 0.000 \\
\hline UGT (sec) & $5.57 \pm 1.21$ & $4.49 \pm 0.76$ & -19.3 & 0.000 \\
\hline HG (kg) & $22.7 \pm 9.8$ & $23.51 \pm 8.32$ & 3.8 & 0.597 \\
\hline $\mathrm{KET}(\mathrm{Nm})$ & $75.34 \pm 18.56$ & $78.49 \pm 15.23$ & 5.5 & 0.179 \\
\hline $\mathrm{KEM}(\mathrm{Nm})$ & $111.52 \pm 41.07$ & $120.40 \pm 39.36$ & $|4.6|$ & 0.138 \\
\hline \multicolumn{5}{|c|}{ Land group $(n=17)$} \\
\hline $\mathrm{BS}(\mathrm{cm})$ & $-4.9 \pm 6.5$ & $-3.47 \pm 8.35$ & 29.0 & 0.533 \\
\hline SR $(\mathrm{cm})$ & $5.3 \pm 12.2$ & $|3.53 \pm| 1.5 \mid$ & 153.7 & 0.003 \\
\hline UGT (sec) & $6.08 \pm 2.06$ & $5.31 \pm 2.08$ & -12.6 & 0.013 \\
\hline HG (kg) & $18.7 \pm 8.4$ & $23.55 \pm 9.14$ & 26.1 & 0.045 \\
\hline KET (Nm) & $67.06 \pm 21.34$ & $68.73 \pm 19.33$ & -1.0 & 0.702 \\
\hline $\mathrm{KEM}(\mathrm{Nm})$ & $101.16 \pm 47.95$ & $103.87 \pm 53.13$ & -0.7 & 0.891 \\
\hline \multicolumn{5}{|c|}{ Control group $(n=19)$} \\
\hline $\mathrm{BS}(\mathrm{cm})$ & $-4.7 \pm 12$ & $-4.45 \pm 9.60$ & 5.3 & 0.326 \\
\hline $\mathrm{SR}(\mathrm{cm})$ & $8.9 \pm 7.8$ & $10.37 \pm 7.62$ & 16.5 & 0.103 \\
\hline UGT (sec) & $5.11 \pm 0.79$ & $5.28 \pm 1.02$ & 3.3 & 0.304 \\
\hline HG (kg) & $20.7 \pm 9.8$ & $23.54 \pm 10.65$ & 13.9 & 0.096 \\
\hline KET (Nm) & $74.23 \pm 20.34$ & $64.41 \pm 22.96$ & -14.4 & 0.000 \\
\hline $\mathrm{KEM}(\mathrm{Nm})$ & $113.28 \pm 57.34$ & $84.60 \pm 40.23$ & -25.5 & 0.006 \\
\hline
\end{tabular}

Abbreviations: BS, back scratch test; SR, sit-and-reach test; UGT, 8-foot Up and Go test; HG, hand grip test; KET, isotonic leg extension strength; KEM, isometric leg extension strength; $\Delta$, difference between $T_{1}$ and $T_{0}$.

Post hoc analyses indicated that AG had the largest improvement in the UGT compared to the other two groups (AG $<$ LG, $P=0.012$; AG $<$ CG, $P<0.001$ ) after 6 months of exercise (Figure 2); also, in LG, UGT improvement was greater than that of $\mathrm{CG}(\mathrm{LG}<\mathrm{CG}, P=0.003)$. Yet in isotonic and isometric knee-extension assessments, CG showed declines, with changes that were significantly different in comparison with the other two groups (KET CG $<\mathrm{AG}$, $P<0.001$ and $\mathrm{CG}<\mathrm{LG}, P=0.007$; KEM CG $<\mathrm{AG}$, $P=0.002$ and $\mathrm{CG}<\mathrm{LG}, P=0.005)$. No differences were found between AG and LG. With regard to body composition, AG diminished total FM and trunk FFM only with respect to $\mathrm{CG}$ (total FM, AG $<\mathrm{CG}, P<0.001$; trunk FM, $\mathrm{AG}<\mathrm{CG}, P=0.005)$. Finally, $\mathrm{LG}$ increased trunk and total FFM compared with AG and CG (trunk FFM, LG $>$ AG, $P=0.006, \mathrm{LG}>\mathrm{CG}, P=0.012$; total FFM, LG $>$ AG, $P=0.005, \mathrm{LG}>\mathrm{CG}, P=0.011)$.

\section{Discussion}

The aim of our study was to evaluate the effects of a 6-month twice-weekly water-based physical activity program in a group of healthy elderly individuals, in comparison with a land-based exercise group and a control group. Our data suggest that both water- and land-based activities, performed at the same RPE, can be beneficial in maintaining strength and in increasing lower-body flexibility. Further, aquatic exercise appears an effective means to improve dynamic balance. On the whole, these results support the use of warm-water exercise among the effective training modalities employed in the elderly population.

A novel approach of this manuscript is based on the use of warm water as an exercise setting for healthy elderly. This choice results in agreement with the need for a higher water temperature for water exercise in older subjects with respect to their younger counterparts, as suggested by Barbosa et al. ${ }^{26}$ To our knowledge, there are only two former manuscripts evaluating the effect of a water-based program in hot spa water, ${ }^{27,28}$ however, they investigated only balance and fall risk reduction, while our study is the first to analyze also the effect on strength and physical performance. Another novel approach of our investigation is the use of recent imaging techniques to assess body composition not yet widely reported ${ }^{18}$ in the current literature. ${ }^{14}$ Another important consideration is that this study is the first comparing the effects of similar exercise protocols, carried out in two different environments. In fact, to our knowledge, no other research has compared exercise protocols performed at the same exercise intensity (RPE) in two different settings (water versus land) on healthy elderly subjects. Only two studies compared different exercise modalities: an aquatic group with a walking group, ${ }^{29}$ and progressive resistance training with progressive aerobic aquatic training. ${ }^{30}$

Overall lower-body strength was maintained in AG and LG after the 6-month physical activity intervention. Existing literature provides a few papers evaluating lower-limb strength after an aquatic exercise protocol in the elderly. ${ }^{31-34}$ Katsura et $\mathrm{al}^{31}$ and Graef et al ${ }^{133}$ described the strength/exercise intensity through the RPE scale, while Takeshima et $\mathrm{l}^{32}$ instructed participants to move through a full range of motion and as fast as possible, for each repetition on each set. Tsourlou et $\mathrm{al}^{34}$ carried out resistance training using water-resistance products, keeping the rhythm with music pace. Compared to our data, these last three studies ${ }^{32-34}$ showed greater and statistically significant improvements. However, both Takeshima et $\mathrm{al}^{32}$ and Tsourlou et a ${ }^{34}$ used specific devices able to increase the drag force, probably reaching a higher strength intensity. In addition, those protocols were set with three exercise sessions per week versus our two sessions. On the contrary, in the Katsura et al study, ${ }^{31}$ the duration of the intervention was probably too short ( 8 weeks) to yield a significant increase in isokinetic knee-extension strength. 
Table 4 Effect of the exercise program on body composition (mean \pm standard deviation)

\begin{tabular}{|c|c|c|c|c|}
\hline & Pre $\left(\mathrm{T}_{0}\right)$ & Post $\left(T_{1}\right)$ & $\Delta \%$ & $\mathbf{P}$ \\
\hline \multicolumn{5}{|l|}{ Aquatic group $(n=17)$} \\
\hline Body mass $(\mathrm{kg})$ & $69.7 \pm 13.4$ & $68.8 \pm 13.6$ & -1.3 & 0.041 \\
\hline $\mathrm{BMI}\left(\mathrm{kg} \cdot \mathrm{m}^{-2}\right)$ & $26.6 \pm 2.8$ & $26.2 \pm 2.7$ & -1.4 & 0.037 \\
\hline Appendicular FM (g) & $824 I .5 \pm 24 \mid 0.1$ & $8022.2 \pm 2453.6$ & -2.7 & 0.216 \\
\hline Trunk FM (g) & I I $003.5 \pm 4032.2$ & $10417.2 \pm 3692.2$ & -5.3 & 0.011 \\
\hline Total FM (g) & $20076.3 \pm 5699.0$ & $19276.0 \pm 5427.1$ & -4.0 & 0.039 \\
\hline Appendicular FFM (g) & $19444.9 \pm 4379.6$ & $19170.3 \pm 4354.1$ & -1.4 & 0.493 \\
\hline $\operatorname{ASMM}\left(\mathrm{kg} \cdot \mathrm{m}^{-2}\right)$ & $7.40 \pm 1.09$ & $7.29 \pm 1.12$ & -1.5 & 0.764 \\
\hline Trunk FFM (g) & $23381.8 \pm 5110.4$ & $23360.0 \pm 5058.2$ & -0.1 & 0.912 \\
\hline Total FFM (g) & $46005.7 \pm 9619.0$ & $45732.8 \pm 9581.7$ & -0.6 & 0.295 \\
\hline Radius muscle density $\left(\mathrm{mg} \cdot \mathrm{m}^{-3}\right)$ & $74.30 \pm 2.70$ & $74.97 \pm 2.44$ & 0.9 & 0.232 \\
\hline Radius muscle area $\left(\mathrm{mm}^{2}\right)$ & $3394.4 \pm 735.0$ & $3510.0 \pm 750.2$ & 3.4 & 0.167 \\
\hline Radius fat area $\left(\mathrm{mm}^{2}\right)$ & $1082.7 \pm 352.5$ & $983.5 \pm 361.2$ & -9.2 & 0.049 \\
\hline Radius total area $\left(\mathrm{mm}^{2}\right)$ & $4832.1 \pm 804.6$ & $4856.7 \pm 839.7$ & 0.5 & 0.174 \\
\hline Tibia muscle density $\left(\mathrm{mg} \cdot \mathrm{m}^{-3}\right)$ & $70.86 \pm 1.83$ & $72.14 \pm 1.76$ & 1.8 & 0.014 \\
\hline Tibia muscle area $\left(\mathrm{mm}^{2}\right)$ & $6926.7 \pm 1405.3$ & $6959.3 \pm 1410.9$ & 0.5 & 0.991 \\
\hline Tibia fat area $\left(\mathrm{mm}^{2}\right)$ & $1826.4 \pm 865.9$ & $1879.0 \pm 842.2$ & 2.9 & 0.885 \\
\hline Tibia total area $\left(\mathrm{mm}^{2}\right)$ & $9479.6 \pm 946.9$ & $9563.7 \pm 1060.3$ & 0.9 & 0.844 \\
\hline \multicolumn{5}{|l|}{ Land group $(n=17)$} \\
\hline Body mass $(\mathrm{kg})$ & $69.1 \pm 13.9$ & $68.9 \pm 13.4$ & -0.3 & 0.657 \\
\hline $\mathrm{BMI}\left(\mathrm{kg} \cdot \mathrm{m}^{-2}\right)$ & $25.7 \pm 3.5$ & $25.7 \pm 3.4$ & -0.2 & 0.734 \\
\hline Appendicular FM (g) & $7490.8 \pm 2898.5$ & $7158.1 \pm 3507.1$ & -4.4 & 0.827 \\
\hline Trunk FM (g) & $9827.3 \pm 3620.0$ & $9640.7 \pm 3869.1$ & -1.9 & 0.418 \\
\hline Total FM (g) & $18167 . \mid \pm 5956.5$ & $|8| 12.8 \pm 6425.8$ & -0.3 & 0.793 \\
\hline Appendicular FFM (g) & $20055.3 \pm 4996.1$ & $18293.3 \pm 6751.7$ & -8.8 & 0.444 \\
\hline $\operatorname{ASMM}\left(\mathrm{kg} \cdot \mathrm{m}^{-2}\right)$ & $7.39 \pm 1.25$ & $7.28 \pm 1.17$ & -1.6 & 0.498 \\
\hline Trunk FFM (g) & $23783.6 \pm 5548.0$ & $23966.8 \pm 5544.6$ & 0.8 & 0.014 \\
\hline Total FFM (g) & $46621.0 \pm 10727.6$ & $47040.6 \pm 10827.8$ & 0.9 & 0.009 \\
\hline Radius muscle density $\left(\mathrm{mg} \cdot \mathrm{m}^{-3}\right)$ & $74.60 \pm 2.63$ & $75.85 \pm 2.22$ & 1.7 & 0.040 \\
\hline Radius muscle area $\left(\mathrm{mm}^{2}\right)$ & $3483.3 \pm 984.7$ & $3587.2 \pm 975.2$ & 3.0 & 0.120 \\
\hline Radius fat area $\left(\mathrm{mm}^{2}\right)$ & $1005.6 \pm 531.0$ & $1093.5 \pm 612.2$ & 8.7 & 0.728 \\
\hline Radius total area $\left(\mathrm{mm}^{2}\right)$ & $4868.6 \pm 1194.3$ & $5046.3 \pm 1034.5$ & 3.6 & 0.523 \\
\hline Tibia muscle density $\left(\mathrm{mg} \cdot \mathrm{m}^{-3}\right)$ & $71.90 \pm 3.29$ & $72.07 \pm 3.79$ & 0.2 & 0.966 \\
\hline Tibia muscle area $\left(\mathrm{mm}^{2}\right)$ & $6825.7 \pm 1590.5$ & $6986.6 \pm 1606.2$ & 2.4 & 0.402 \\
\hline Tibia fat area $\left(\mathrm{mm}^{2}\right)$ & $1979.0 \pm 1069.3$ & $2163.1 \pm 1110.1$ & 9.3 & 0.346 \\
\hline Tibia total area $\left(\mathrm{mm}^{2}\right)$ & $9583.9 \pm 1708.1$ & $9938.5 \pm 1639.7$ & 3.7 & 0.025 \\
\hline \multicolumn{5}{|l|}{ Control group $(n=19)$} \\
\hline Body mass $(\mathrm{kg})$ & $65.4 \pm 10.8$ & $65.2 \pm 9.6$ & -0.3 & 0.315 \\
\hline $\mathrm{BMI}\left(\mathrm{kg} \cdot \mathrm{m}^{-2}\right)$ & $24.4 \pm 2.6$ & $24.1 \pm 2.4$ & -1.2 & 0.103 \\
\hline Appendicular FM (g) & $7969.1 \pm 2983.1$ & $8301.8 \pm 2790.6$ & 4.3 & 0.074 \\
\hline Trunk FM (g) & $9218.0 \pm 2688.2$ & $9511.8 \pm 2568.0$ & 3.2 & 0.115 \\
\hline Total FM (g) & $18144.3 \pm 5101.0$ & $20162.5 \pm 4412.8$ & 11.1 & 0.000 \\
\hline Appendicular FFM (g) & $18701.6 \pm 5008.9$ & $18547.9 \pm 5064.1$ & -0.8 & 0.117 \\
\hline $\operatorname{ASMM}\left(\mathrm{kg} \cdot \mathrm{m}^{-2}\right)$ & $6.86 \pm 1.27$ & $6.79 \pm 1.27$ & -0.9 & 0.315 \\
\hline Trunk FFM (g) & $22469.7 \pm 4512.0$ & $22015.6 \pm 4363.4$ & -2.0 & 0.047 \\
\hline Total FFM (g) & $44286.5 \pm 9910.8$ & $43376.5 \pm 9676.9$ & -2.1 & 0.003 \\
\hline Radius muscle density $\left(\mathrm{mg} \cdot \mathrm{m}^{-3}\right)$ & $75.19 \pm 2.76$ & $75.14 \pm 2.41$ & -0.1 & 0.891 \\
\hline Radius muscle area $\left(\mathrm{mm}^{2}\right)$ & $3281.6 \pm 925.5$ & $3239.6 \pm 1013.1$ & -1.3 & 0.404 \\
\hline Radius fat area $\left(\mathrm{mm}^{2}\right)$ & $1169.9 \pm 463.4$ & || $29.1 \pm 44 \mid .0$ & -3.5 & 0.085 \\
\hline Radius total area $\left(\mathrm{mm}^{2}\right)$ & $4774.8 \pm 788.1$ & $4733.4 \pm 842.6$ & -0.9 & 0.163 \\
\hline Tibia muscle density $\left(\mathrm{mg} \cdot \mathrm{m}^{-3}\right)$ & $72.47 \pm 2.94$ & $72.93 \pm 2.23$ & 0.6 & 0.412 \\
\hline Tibia muscle area $\left(\mathrm{mm}^{2}\right)$ & $6944.7 \pm 1817.7$ & $7093.5 \pm 1833.4$ & 2.1 & 0.105 \\
\hline Tibia fat area $\left(\mathrm{mm}^{2}\right)$ & $2091.4 \pm 936.3$ & $2172.2 \pm 831.9$ & 3.9 & 0.527 \\
\hline Tibia total area $\left(\mathrm{mm}^{2}\right)$ & $9755.2 \pm 1482.8$ & $10014.8 \pm 1539.8$ & 2.7 & 0.014 \\
\hline
\end{tabular}

Abbreviations: BMI, body mass index; FM, fat mass; FFM, fat free mass; ASMM, appendicular skeletal muscle mass; $\Delta$, difference between $T_{1}$ and $T_{0}$. 


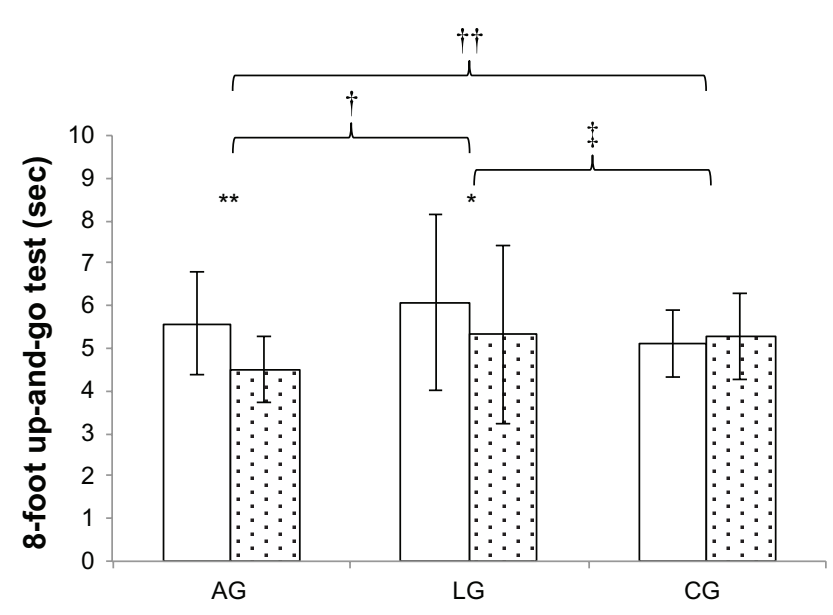

Figure 2 Dynamic balance at baseline (white columns) and after six months (dotted columns).

Notes: *Indicates a statistically significant difference $(P<0.05)$; **indicates $P$ value $<0.01$; tindicates a between-group statistically significant difference $(P<0.01)$ while *indicates between-group significance with $P$ value $<0.05$; ${ }^{+1}$ intra groups indicates statistically significant difference (ANOVA).

Abbreviations: AG, aquatic group; LG, land group; CG, control group.

Grip strength was investigated as a surrogate of overall muscle strength as well as its role in predicting disability and mobility limitation. ${ }^{35,36}$ After the training period, hand-grip strength did not show any significant increase in AG. Three other studies performed similar measures, in some cases observing improvements, ${ }^{34,37}$ while in another not. ${ }^{38}$ Similarly to lower-body strength, it could be hypothesized that the absence of specific training might have determined the lack of effect on hand-grip performance. Interestingly, grip strength increased by $26 \%$ in LG, with a concomitant increase in forearm muscle density. This improvement could be explained by greater involvement of forearm muscles during LG activity due to gravity, although we do not have a certain explanation. Considering that higher muscle density, reflecting a lower fat infiltration into muscle mass, ${ }^{20}$ could positively affect muscle strength, ${ }^{39}$ we could hypothesize a possible relationship between LG hand-grip and forearm muscle density.

AG increased both shoulder range of motion and lower-body flexibility, although the clinical improvement in upper-body flexibility appears of limited importance..$^{15}$ Other investigations carried out the same tests to measure upper- and lower-body flexibility, eg, Bocalini et al ${ }^{29}$ observed an improvement in the upper body by $40 \%$. On the contrary, there is a discrepancy in the literature about lower-body flexibility. Some studies did not show any difference after the aquatic exercise protocol, ${ }^{32,38}$ whereas others highlighted large positive changes. ${ }^{29,31,34,37}$ None of these cited studies specified the type and the intensity maintained during flexibility exercises, and only some papers reported the time (mostly from 7 to 20 minutes) dedicated to each exercise session. ${ }^{29,30,32,37,38}$ Despite this lack of adequate descriptions, we could hypothesize that the greater difficulty in training lower-body flexibility in water (ie, to perform exercises where subjects reach their maximal degrees of range of motion) could be responsible for the observed variability. Our data suggest the feasibility and the potential usefulness of training performed in warm water to improve body flexibility, but not its greater efficacy, given the lack of a significant improvement with respect to the other exercise modality (LG).

Performance in UGT improved in AG and LG. The beforeand-after training effect detected by post hoc analyses showed a gain in $A G$ that was significantly greater with respect to both the other two groups (LG and CG). Since the protocol did not provide any direct intervention to enhance dynamic balance, the result suggests that warm-water intervention or the water setting could be a useful exercise modality or environment to develop dynamic balance in the elderly, apparently more effective than land-based exercise. Other authors observed similar outcomes..$^{27,29,31,40}$ These results could support the hypothesis that the water environment can create positive adaptations in dynamic balance, possibly through continuous instability applied to the subject exercising in an upright position. Considering that our exercise protocol did not include any specific balance exercise, this could suggest that subjects can be trained for specific conditioning (aerobic or cardiovascular, strength, flexibility, etc) while obtaining concomitant benefits in dynamic balance.

A further explanation could arise from the observation that calf muscle strength after the training period was significantly increased only in AG. This effect can probably be best explained by the underwater positioning on toes, which makes balance maintenance easier. ${ }^{19}$ This result could support the role of muscle strength, suggesting the hypothesis of a correlation between leg muscle density, muscle strength, and dynamic balance (expressed by the faster time in the UGT), as previously suggested by MacIntyre et $\mathrm{al}^{41}$ in stroke survivors with subacute chronic lower-limb hemiparesis. A recent analysis arising from a review article by Granacher et $\mathrm{al}^{42}$ supports a similar assumption, observing that the improvement in gait speed (a possible determinant of performance in the UGT) is related to measures of isometric/dynamic strength and power. The lack of data regarding calf muscle strength prevents us from confirming this hypothesis.

FM decreased by $4 \%$ in AG, with the trunk area showing the highest reductions. In addition, dominant forearm fat decreased by $9.16 \%$. The effect of training on total and trunk fat-mass reduction in AG was significantly greater 
compared to that observed in CG. A previous study, involving water-based activities, measured the sum of skin folds, finding a reduction in FM by $8 \%,{ }^{32}$ while another ${ }^{38}$ did not show any change. Also, Ruoti et al, ${ }^{43}$ using underwater weighting, did not detect any reduction in FM.

Conversely, land-based physical activity seemed more useful to increase FFM compared to CG. However, despite a statistical 1\% improvement in muscle mass, we believe that this change cannot be considered clinically meaningful. ${ }^{11}$ To our knowledge, the only investigation that measured FFM was that of Tsourlou et al, ${ }^{34}$ showing an increase by $3.4 \%$ after a 24 -week aquatic training. Compared to our $\mathrm{AG}$, where no changes were found in FFM, the effectiveness of this aquatic exercise protocol in increasing FFM may be related to the higher number of weekly training sessions, as well as to the use of waterresistance devices to increase drag resistance. On the whole, despite their importance, food intake and energy balance were never monitored together in any of the existing studies. Future studies should take into account these essential aspects, and possibly evaluate which protocol could be more effective in increasing FFM or promoting weight loss, by means of more advanced techniques of body-composition assessment.

Regarding the safety of the protocols and environment adopted, exercise intensity was never so high as to induce any subject to report significant thermoregulatory distress. Warm water was always well tolerated, and we did not observe any injury or significant adverse effect during the whole training period in either experimental group. These observations, although anecdotal, support the safety and feasibility of similar protocols in a specific setting such as warm-water pools.

A limitation of the study was the rather small sample size. For this reason, these findings should be reported with caution to the elderly population before confirmation is received. An additional criticism arises from the intrinsic characteristics of elderly subjects, who despite being clinically eligible to participate in the exercise program or asymptomatic, may have presented underlying pathologies potentially influencing the final results. Finally, we cannot exclude a disparity between the "objective" exercise intensity in the two environments (water versus land), despite a similar "subjective" intensity of exercise perception (RPE), as suggested by Killgore et al. ${ }^{44}$

\section{Conclusion}

In summary, both warm-water and land-based activities, performed at the same RPE, were beneficial in maintaining strength and in improving lower-body flexibility.
Between protocols, the aquatic exercise appeared a better activity to increase dynamic balance and promote weight loss, although further data are needed to confirm these findings. Warm-water swimming pools and the use of RPE as a method of exercise monitoring should be considered suitable tools to enhance physical fitness in healthy elderly subjects.

\section{Acknowledgments}

The authors thank all the family physicians who meticulously participated in the recruitment process. We also thank Mrs Zanettin, Mr Fioraso, and Mr Mattiazzo, directors of the thermal accommodations, who kindly offered their facilities in order to develop this investigation. This research was supported by the European Social Fund, grant 2105/101/1/722/2009.

\section{Disclosure}

The authors have no conflict of interest with regard to this research.

\section{References}

1. Baumgartner RN, Koehler KM, Gallagher D, et al. Epidemiology of sarcopenia among the elderly in New Mexico. Am J Epidemiol. 1998;147(8):755-763.

2. Taaffe DR. Sarcopenia - exercise as a treatment strategy. Aust Fam Physician. 2006;35(3):130-134.

3. Paddon-Jones D, Rasmussen BB. Dietary protein recommendations and the prevention of sarcopenia. Curr Opin Clin Nutr Metab Care. 2009;12(1):86-90.

4. Borst SE. Interventions for sarcopenia and muscle weakness in older people. Age Ageing. 2004;33(6):548-555.

5. Doherty TJ. Invited review: Aging and sarcopenia. J Appl Physiol. 2003;95(4):1717-1727.

6. Cruz-Jentoft AJ, Baeyens JP, Bauer JM, et al. Sarcopenia: European consensus on definition and diagnosis: Report of the European Working Group on Sarcopenia in Older People. Age Ageing. 2010;39(4): 412-423.

7. Laurent M, Daline T, Malika B, et al. Training-induced increase in nitric oxide metabolites in chronic heart failure and coronary artery disease: an extra benefit of water-based exercises? Eur J Cardiovasc Prev Rehabil. 2009;16(2):215-221.

8. Tokmakidis SP, Spassis AT, Volaklis KA. Training, detraining and retraining effects after a water-based exercise program in patients with coronary artery disease. Cardiology. 2008;111(4):257-264.

9. Tomas-Carus P, Gusi N, Hakkinen A, Hakkinen K, Raimundo A, Ortega-Alonso A. Improvements of muscle strength predicted benefits in HRQOL and postural balance in women with fibromyalgia: an 8-month randomized controlled trial. Rheumatology (Oxford). 2009;48(9):1147-1151.

10. Munguia-Izquierdo D, Legaz-Arrese A. Assessment of the effects of aquatic therapy on global symptomatology in patients with fibromyalgia syndrome: a randomized controlled trial. Arch Phys Med Rehabil. 2008;89(12):2250-2257.

11. Waller B, Lambeck J, Daly D. Therapeutic aquatic exercise in the treatment of low back pain: a systematic review. Clin Rehabil. 2009;23(1): 3-14.

12. Dundar U, Solak O, Yigit I, Evcik D, Kavuncu V. Clinical effectiveness of aquatic exercise to treat chronic low back pain: a randomized controlled trial. Spine (Phila Pa 1976). 2009;34(14):1436-1440. 
13. Tolomio S, Ermolao A, Lalli A, Zaccaria M. The effect of a multicomponent dual-modality exercise program targeting osteoporosis on bone health status and physical function capacity of postmenopausal women. J Women Aging. 2010;22(4):241-254.

14. Bergamin M, Zanuso S, Alvar BA, Ermolao A, Zaccaria M. Is waterbased exercise training sufficient to improve physical fitness in the elderly? Eur Rev Aging Phys A. Oct 2012;9(2):129-141.

15. Hall J, Swinkels A, Briddon J, McCabe CS. Does aquatic exercise relieve pain in adults with neurologic or musculoskeletal disease? A systematic review and meta-analysis of randomized controlled trials. Arch Phys Med Rehabil. 2008;89(5):873-883.

16. Rikli RE, Jones CJ. Development and validation of a functional fitness test for a community-residing adults. J Aging Phys Act. 1999;7(2): 129-161.

17. Salamone LM, Fuerst T, Visser M, et al. Measurement of fat mass using DEXA: a validation study in elderly adults. JAppl Physiol. 2000;89(1): 345-352.

18. Visser M, Fuerst T, Lang T, Salamone L, Harris TB. Validity of fanbeam dual-energy X-ray absorptiometry for measuring fat-free mass and leg muscle mass. Health, Aging, and Body Composition Study Dual-Energy X-ray Absorptiometry and Body Composition Working Group. J Appl Physiol. 1999;87(4):1513-1520.

19. Mitsiopoulos N, Baumgartner RN, Heymsfield SB, Lyons W, Gallagher D, Ross R. Cadaver validation of skeletal muscle measurement by magnetic resonance imaging and computerized tomography. J Appl Physiol. 1998;85(1):115-122.

20. Goodpaster BH, Kelley DE, Thaete FL, He J, Ross R. Skeletal muscle attenuation determined by computed tomography is associated with skeletal muscle lipid content. J Appl Physiol. 2000;89(1):104-110.

21. Larson-Meyer DE, Smith SR, Heilbronn LK, et al. Muscle-associated triglyceride measured by computed tomography and magnetic resonance spectroscopy. Obesity (Silver Spring). 2006;14(1):73-87.

22. Butner KL, Creamer KW, Nickols-Richardson SM, Clark SF, Ramp WK, Herbert WG. Fat and muscle indices assessed by pQCT: relationships with physical activity and type 2 diabetes risk. J Clin Densitom. 2012;15(3):355-361.

23. Borg G. Perceived exertion as an indicator of somatic stress. Scand J Rehabil Med. 1970;2(2):92-98.

24. Chodzko-Zajko WJ, Proctor DN, Fiatarone Singh MA, et al. American College of Sports Medicine position stand. Exercise and physical activity for older adults. Med Sci Sports Exerc. 2009;41(7):1510-1530.

25. Cooper KH. A means of assessing maximal oxygen intake. Correlation between field and treadmill testing. JAMA. 1968;203(3):201-204.

26. Barbosa TM, Marinho DA, Reis VM, Silva AJ, Bragada JA. Physiological assessment of head-out aquatic exercises in healthy subjects: a qualitative review. J Sports Sci Med. 2009;8:178-189.

27. Berger L, Klein C, Commandeur M. Evaluation of the immediate and midterm effects of mobilization in hot spa water on static and dynamic balance in elderly subjects. Ann Readapt Med Phys. 2008;51(2):84-95.

28. Hale LA, Waters D, Herbison P. A randomized controlled trial to investigate the effects of water-based exercise to improve falls risk and physical function in older adults with lower-extremity osteoarthritis. Arch Phys Med Rehabil. 2012;93(1):27-34.
29. Bocalini DS, Serra AJ, Murad N, Levy RF. Water- versus land-based exercise effects on physical fitness in older women. Geriatr Gerontol Int. 2008;8(4):265-271.

30. Vale RG, de Oliveira RD, Pernambuco CS, de Meneses YP, Novaes Jda S, de Andrade Ade F. Effects of muscle strength and aerobic training on basal serum levels of IGF-1 and cortisol in elderly women. Arch Gerontol Geriatr. 2009;49(3):343-347.

31. Katsura Y, Yoshikawa T, Ueda SY, et al. Effects of aquatic exercise training using water-resistance equipment in elderly. Eur J Appl Physiol. 2010;108(5):957-964

32. Takeshima N, Rogers ME, Watanabe E, et al. Water-based exercise improves health-related aspects of fitness in older women. $\mathrm{Med} \mathrm{Sci}$ Sports Exerc. 2002;34(3):544-551.

33. Graef FI, Pinto RS, Alberton CL, de Lima WC, Kruel LF. The effects of resistance training performed in water on muscle strength in the elderly. J Strength Cond Res. 2010;24(11):3150-3156.

34. Tsourlou T, Benik A, Dipla K, Zafeiridis A, Kellis S. The effects of a twenty-four-week aquatic training program on muscular performance in healthy elderly women. J Strength Cond Res. 2006;20(4): 811-818.

35. Rantanen T, Guralnik JM, Foley D, et al. Midlife hand grip strength as a predictor of old age disability. JAMA. 1999;281(6):558-560.

36. Shinkai S, Watanabe S, Kumagai S, et al. Walking speed as a good predictor for the onset of functional dependence in a Japanese rural community population. Age Ageing. 2000;29(5):441-446.

37. Cancela Carral JM, Ayàn Pérez C. Effects of high-intensity combined training on women over 65. Gerontology. 2007;53(6):340-346.

38. Taunton JE, Rhodes EC, Wolski LA, et al. Effect of land-based and water-based fitness programs on the cardiovascular fitness, strength and flexibility of women aged 65-75 years. Gerontology. 1996;42(4): 204-210.

39. Delmonico MJ, Harris TB, Lee JS, et al. Alternative definitions of sarcopenia, lower extremity performance, and functional impairment with aging in older men and women. J Am Geriatr Soc. 2007;55(5): 769-774.

40. Resende SM, Rassi CM, Viana FP. Effect of hydrotherapy in balance and prevention of falls among elderly women. Rev Bras Fisioter. 2008;12(1):57-63.

41. MacIntyre NJ, Rombough R, Brouwer B. Relationship between calf muscle density and muscle strength, mobility and bone status in the stroke survivors with subacute and chronic lower limb hemiparesis. J Musculoskelet Neuronal Interact. 2010;10(4): 249-255.

42. Granacher U, Muehlbauer T, Gruber M. A qualitative review of balance and strength performance in healthy older adults: impact for testing and training. J Aging Res. 2012;2012:708905.

43. Ruoti RG, Troup JT, Berger RA. The effects of nonswimming water exercises on older adults. J Orthop Sports Phys Ther. Mar 1994; 19(3):140-145.

44. Killgore GL, Coste SC, SE OM, Konnecke CJ. A comparison of the physiological exercise intensity differences between shod and barefoot submaximal deep-water running at the same cadence. J Strength Cond Res. Dec 2010;24(12):3302-3312.
Clinical Interventions in Aging

\section{Publish your work in this journal}

Clinical Interventions in Aging is an international, peer-reviewed journal focusing on evidence-based reports on the value or lack thereof of treatments intended to prevent or delay the onset of maladaptive correlates of aging in human beings. This journal is indexed on PubMed Central, MedLine, the American Chemical Society's 'Chemical Abstracts

\section{Dovepress}

Service' (CAS), Scopus and the Elsevier Bibliographic databases. The manuscript management system is completely online and includes a very quick and fair peer-review system, which is all easy to use. Visit http://www.dovepress.com/testimonials.php to read real quotes from published authors. 